ICRR-Report-340-95-6

LBL-37715

UT-719

hep-ph/???????

\title{
Constraint on the Reheating Temperature from the Decay of the Polonyi Field of
}

\author{
M. Kawasaki ${ }^{a}$, T. Moroi $^{b}$ and T. Yanagida ${ }^{c, d}$ \\ ${ }^{a}$ Institute for Cosmic Ray Research, University of Tokyo, Tokyo 188, JAPAN \\ ${ }^{b}$ Theoretical Physics Group, Lawrence Berkeley Laboratory, University of California, \\ Berkeley, CA 94720 , U.S.A. \\ ${ }^{c}$ Department of Physics, University of Tokyo, Tokyo 133, JAPAN \\ ${ }^{d}$ Institute for Theoretical Physics, University of California, Santa Barbara, CA 93106, \\ U.S.A.
}

\begin{abstract}
We study the Polonyi problem in the framework of no-scale type supergravity models. We show that the lightest superparticle (LSP) produced in the decay of the Polonyi field may contribute too much to the present density of the universe. By requiring that LSP should not overclose the universe, we obtain a stringent constraint on the reheating temperature after the decay of the Polonyi field. We calculate the LSP density with physical parameters obtained by solving renormalization group equations in the minimal supersymmetric $\mathrm{SU}(5)$ model and find that the reheating temperature should be greater than about $100 \mathrm{MeV}$ which corresponds to $O(100) \mathrm{TeV}$ of the Polonyi mass.
\end{abstract}

${ }^{*}$ This work was supported by the Director, Office of Energy Research, Office of High Energy and Nuclear Physics, Division of High Energy Physics of the U.S. Department of Energy under Contract DE-AC03-76SF00098. 


\section{Disclaimer}

This document was prepared as an account of work sponsored by the United States Government. While this document is believed to contain correct information, neither the United States Government nor any agency thereof, nor The Regents of the University of California, nor any of their employees, makes any warranty, express or implied, or assumes any legal liability or responsibility for the accuracy, completeness, or usefulness of any information, apparatus, product, or process disclosed, or represents that its use would not infringe privately owned rights. Reference herein to any specific commercial products process, or service by its trade name, trademark, manufacturer, or otherwise, does not necessarily constitute or imply its endorsement, recommendation, or favoring by the United States Government or any agency thereof, or The Regents of the University of California. The views and opinions of authors expressed herein do not necessarily state or reflect those of the United States Government or any agency thereof, or The Regents of the University of California.

Lawrence Berkeley Laboratory is an equal opportunity employer. 


\section{Introduction}

The Polonyi problem [1], 2] is one of the most serious problems in models based on the $N=1$ supergravity [3]. In a wide class of supergravity models, the Polonyi field $\phi$, which is a scalar field related to the supersymmetry (SUSY) breaking, has a mass $m_{\phi}$ of the order of the gravitino mass. During inflation, $\phi$ takes an amplitude of the order of the gravitational scale $M \equiv M_{\mathrm{pl}} / \sqrt{8 \pi} \simeq 2.4 \times 10^{18} \mathrm{GeV}$. After the inflation, the condensation of $\phi$ starts to oscillate when the expansion rate of the universe, $H$, becomes comparable to $m_{\phi}$ and finally decays into particles in the observable sector. Since the interactions of the Polonyi field are suppressed by powers of $M^{-1}$, the decay rate of the Polonyi field, $\Gamma_{\phi}$, is very small as

$$
\Gamma_{\phi} \sim N \frac{m_{\phi}^{3}}{M_{\mathrm{pl}}^{2}},
$$

where $N$ is the number of the decay modes. (In the following calculations, we take $N=100$.) Therefore, the Polonyi field is expected to decay when the temperature of the universe becomes very low. The reheating temperature $T_{R}$ due to the decay of the Polonyi field is given by

$$
T_{R} \sim 1 \mathrm{MeV}\left(\frac{m_{\phi}}{10 \mathrm{TeV}}\right)^{3 / 2} .
$$

This causes serious cosmological difficulties; namely the Polonyi field may destroy the great success of the big-bang nucleosynthesis (BBN), and the entropy production due to its decay may dilute the baryon number of the universe too much.

In the previous works 田, 鴫 it has been pointed out that the Polonyi problem can be solved if the gravitino mass $m_{3 / 2}$ (which is the same order of the Polonyi mass) is larger than $O(10) \mathrm{TeV}$ in order to hasten the decay of the Polonyi field. Thus, it is desirable to raise the gravitino mass while keeping the effective SUSY breaking scale in the observable sector at $O(100) \mathrm{GeV}$. In no-scale type supergravity models, such mass hierarchy is realized [G] and hence the no-scale type supergravity model with $m_{3 / 2} \gtrsim O(10) \mathrm{TeV}$ has been suggested as an attractive solution to the Polonyi problem.

In reference [5], however, it has been also pointed out that the mass density of the lightest superparticle (LSP) produced by the decay of the Polonyi field may overclose our universe if LSP is stable. As we will see below, the mass density of LSP increases as the reheating temperature $T_{R}$ due to the decay of the Polonyi field decreases. Therefore, a lowerbound on $T_{R}$ is derived requiring that the present mass density of LSP should not exceed the critical density of the universe $\rho_{c}$. In this letter, we obtain the lowerbound on $T_{R}$ in the framework of the minimal SUSY SU(5) model with no-scale type boundary 
conditions on the SUSY breaking parameters.

\section{The Model}

Before starting cosmological arguments, let us first describe our basic assumptions. We consider the minimal SUSY SU(5) model with no-scale type boundary conditions. This model has three types of Higgs field; $H(\mathbf{5})$ and $\bar{H}\left(\mathbf{5}^{*}\right)$ which contain flavor Higgses $H_{f}$ and $\bar{H}_{f}$, and $\Sigma(\mathbf{2 4})$ whose condensation breaks the $\mathrm{SU}(5)$ group into the gauge group of the minimal SUSY standard model $(\mathrm{MSSM}), \mathrm{SU}(3)_{\mathrm{C}} \times \mathrm{SU}(2)_{\mathrm{L}} \times \mathrm{U}(1)_{\mathrm{Y}}$. For the Higgs sector, the superpotential is given by

$$
W=\frac{1}{3} \lambda \operatorname{tr} \Sigma^{3}+\frac{1}{2} M_{\Sigma} \operatorname{tr} \Sigma^{2}+\kappa \bar{H} \Sigma H+M_{H} \bar{H} H,
$$

where $\lambda$ and $\kappa$ are dimensionless constants, while $M_{\Sigma}$ and $M_{H}$ are mass parameters which are of the order of the grand unified theory (GUT) scale $M_{\mathrm{GUT}}\left(\sim 10^{16} \mathrm{GeV}\right)$. Furthermore, the model also has the soft SUSY breaking terms;

$$
\mathcal{L}_{\text {soft }}=-\frac{1}{3} \lambda A_{\Sigma} \operatorname{tr} \Sigma^{3}-\frac{1}{2} M_{\Sigma} B_{\Sigma} \operatorname{tr} \Sigma^{2}-\kappa A_{H} \bar{H} \Sigma H-M_{H} B_{H} \bar{H} H+\text { h.c. },
$$

where $A_{\Sigma}, B_{\Sigma}, A_{H}$ and $B_{H}$ are SUSY breaking parameters. Minimising the Higgs potential, we find the following stationary point;

$$
\langle\Sigma\rangle=\frac{1}{\lambda}\left\{M_{\Sigma}+2\left(A_{\Sigma}-B_{\Sigma}\right)+O\left(\frac{A_{\Sigma}}{M_{\Sigma}}, \frac{B_{\Sigma}}{M_{\Sigma}}\right)\right\} \times \operatorname{diag}(2,2,2,-3,-3),
$$

where the $\mathrm{SU}(5)$ is broken down to $\mathrm{SU}(3)_{\mathrm{C}} \times \mathrm{SU}(2)_{\mathrm{L}} \times \mathrm{U}(1)_{\mathrm{Y}}$. Regarding this stationary point as the vacuum, we obtain MSSM as the effective theory below the GUT scale $M_{\mathrm{GUT}}$. Here, the masslessness of the flavor Higgses $H_{f}$ and $\bar{H}_{f}$ is achieved by a fine tuning among several parameters; $M_{H}-3 \kappa M_{\Sigma} / \lambda \simeq \mu_{H}$, where $\mu_{H}$ is the SUSY-invariant Higgs mass in MSSM.

In the present model, the parameters in MSSM at the electroweak scale is obtained by solving renormalization group equations (RGEs). Our method is as follows. The boundary conditions on the parameters in the minimal SUSY SU(5) model are given at the gravitational scale $M$. Since we assume the no-scale type supergravity models, all the SUSY breaking parameters except for the gaugino mass vanish at the gravitational scale. From the gravitational scale to the GUT scale, the parameters follow the renormalization group flow derived from RGEs in the minimal SUSY SU(5) model. Then we determine the parameters in MSSM at the GUT scale through an appropriate matching condition 
between the parameters in the SUSY SU(5) model and those in MSSM. Finally, we use RGEs in MSSM from the GUT scale to the electroweak scale in order to obtain the low energy parameters.

As for the matching condition, we have a comment. In the stationary point (5), the mixing soft mass term of the two flavor Higgs bosons, $m_{12}^{2} \bar{H}_{f} H_{f}$, is generated at the tree level, where $m_{12}^{2}$ is given by

$$
m_{12}^{2}\left(M_{\mathrm{GUT}}\right) \simeq\left[\frac{6 \kappa}{\lambda}\left(A_{\Sigma}-B_{\Sigma}\right)\left(A_{H}-B_{\Sigma}\right)-\mu_{H} B_{H} \cdot\right]_{\mu=M_{\mathrm{GUT}}}
$$

Since the mixing mass term depends on unknown parameters, $\lambda$ and $\kappa$ in eq.(3), we regard $m_{12}^{2}$ as a free parameter taking account of the uncertainty of $\lambda$ and $\kappa$ in our analysis. Then, the low energy parameters are essentially determined by the gauge and Yukawa coupling constants and the following three parameters; the supersymmetric Higgs mass $\mu_{H}$, the mixing mass of the two flavor Higgs bosons $m_{12}^{2}$, and the unified gaugino mass. . However, it is more convenient to express these parameters by other physical ones. In fact, one combination of them is constrained so that the flavor Higgs bosons have correct vacuum expectation values; $\left\langle H_{f}\right\rangle^{2}+\left\langle\bar{H}_{f}\right\rangle^{2} \simeq(174 \mathrm{GeV})^{2}$. As the other two physical parameters, we use the mass of LSP, $m_{\mathrm{LSP}}$, and the vacuum angle $\tan \beta \equiv\left\langle H_{f}\right\rangle /\left\langle\bar{H}_{f}\right\rangle$. Thus, once we fix $m_{\mathrm{LSP}}$ and $\tan \beta$, we can determine all the parameters in MSSM.'?

Following the above procedure, we solve the RGEs numerically, and obtain the low energy parameters in MSSM. Then, we determine the mass spectrum and the mixing angles for all superparticles. One remarkable thing is that LSP almost consists of bino $\tilde{B}$ which is the superpartner of the gauge field for $U(1)_{Y}$ if we require that LSP is neutral. Therefore, in our model, the LSP mass $m_{\mathrm{LSP}}$ is essentially equivalent to the bino mass. This fact simplifies the following analysis very much.

\section{Density of LSP}

Now we are in a position to discuss the mass density of LSP produced by the decay of the Polonyi field. The decay of the Polonyi field produces a large number of superparticles, which promptly decay into LSPs. The number density of LSP produced by the decay,

\footnotetext{
${ }^{1}$ In fact, parameters in MSSM slightly depend on the parameters in the SUSY GUT such as $\lambda$, $\kappa$ and so on. In our numerical calculation, we ignore the effects of these parameters on the renormalization group flow.

${ }^{2}$ Yukawa coupling constants are determined so that the fermions have correct masses. The gauge coupling constants are also fixed so that their correct values at the electroweak scale are reproduced.
} 
$n_{\mathrm{LSP}, i}$, is of the same order of that of the Polonyi field $n_{\phi} \equiv \rho_{\phi} / m_{\phi}$ (with $\rho_{\phi}$ being the energy density of the Polonyi field). Just after the decay of the Polonyi field, the yield variable for LSP, $Y_{\mathrm{LSP}}$, which is defined by the ratio of the number density of LSP to the entropy density $s$, is given by

$$
\begin{aligned}
m_{\mathrm{LSP}} Y_{\mathrm{LSP}} & \simeq \frac{\rho_{\phi}}{s} \simeq \frac{m_{\mathrm{LSP}} \rho_{\mathrm{LSP}, i}}{m_{\phi} s} \sim\left(\frac{m_{\mathrm{LSP}} T_{R}}{m_{\phi}}\right) \\
& \sim 10^{-5} \mathrm{GeV}\left(\frac{m_{\mathrm{LSP}}}{100 \mathrm{GeV}}\right)\left(\frac{T_{R}}{1 \mathrm{MeV}}\right)\left(\frac{10 \mathrm{TeV}}{m_{\phi}}\right)
\end{aligned}
$$

where $\rho_{\mathrm{LSP}, i}$ is the mass density of LSP just after the decay of the Polonyi field. If LSP is stable and the pair annihilation of LSP is not effective, $Y_{\mathrm{LSP}}$ is conserved until today. Comparing the ratio given in eq.(17) with the ratio of the critical density $\rho_{c}$ to the present entropy density $s_{0}$,

$$
\frac{\rho_{c}}{s_{0}} \simeq 3.6 \times 10^{-9} h^{2} \mathrm{GeV}
$$

where $h$ is the Hubble constant in units of $100 \mathrm{~km} / \mathrm{sec} / \mathrm{Mpc}$, we see that LSP overcloses the universe in the wide parameter region for $m_{\mathrm{LSP}}, m_{\phi}$ and $T_{R}$ which we are concerned with.

If the pair annihilation of LSP takes place effectively, its abundance is reduced to

$$
\left.\frac{n_{\mathrm{LSP}}}{s} \simeq \frac{H}{s\left\langle\sigma_{\mathrm{ann}} v_{\mathrm{rel}}\right\rangle}\right|_{T=T_{R}}
$$

where $\sigma_{\text {ann }}$ is the annihilation cross section, $v_{\text {rel }}$ is the relative velocity, and $\langle\cdots\rangle$ represents the average over the phase space distribution of LSP. From eq.(9), we obtain a lowerbound on the annihilation cross section,

$$
\left\langle\sigma_{\mathrm{ann}} v_{\mathrm{rel}}\right\rangle \gtrsim 3 \times 10^{-8} h^{-2} \mathrm{GeV}^{-2}\left(\frac{m_{\mathrm{LSP}}}{100 \mathrm{GeV}}\right)\left(\frac{100 \mathrm{MeV}}{T_{R}}\right),
$$

in order that the mass density of LSP does not overclose the universe.

Comparing this bound with the annihilation cross section of LSP, we derive a bound on the reheating temperature by the decay of the Polonyi field. Since LSP is most dominated by bino, it annihilates into fermion pairs. The annihilation cross section is given by [7]

$$
\left\langle\sigma_{\mathrm{ann}} v_{\mathrm{rel}}\right\rangle=a+b\left\langle v^{2}\right\rangle
$$

where $\left\langle v^{2}\right\rangle$ is the average velocity of LSP, and

$$
a \simeq \frac{32 \pi \alpha_{1}^{2}}{27} \frac{m_{t}^{2}}{\left(m_{\tilde{t}_{R}}^{2}+m_{\mathrm{LSP}}^{2}-m_{t}^{2}\right)^{2}}\left(1-\frac{m_{t}^{2}}{m_{\mathrm{LSP}}^{2}}\right)^{1 / 2} \theta\left(m_{\mathrm{LSP}}-m_{t}\right)
$$




$$
b \simeq \frac{8 \pi \alpha_{1}^{2}}{3} \sum_{m_{f} \leq T} Y_{f}^{4}\left\{\frac{m_{\mathrm{LSP}}^{2}}{\left(m_{\mathrm{LSP}}^{2}+m_{\tilde{f}}^{2}\right)^{2}}-\frac{2 m_{\mathrm{LSP}}^{4}}{\left(m_{\mathrm{LSP}}^{2}+m_{\tilde{f}}^{2}\right)^{3}}+\frac{2 m_{\mathrm{LSP}}^{6}}{\left(m_{\mathrm{LSP}}^{2}+m_{\tilde{f}}^{2}\right)^{4}}\right\}
$$

Here, $\alpha_{1}^{2} \equiv g_{1}^{2} / 4 \pi \simeq 0.01$ represents the fine structure constant for $\mathrm{U}(1)_{\mathrm{Y}}, m_{t}$ the topquark mass, $Y_{f}$ the hypercharge of the fermion $f$, and $m_{\tilde{f}}$ the mass of the sfermion $\tilde{f}$. Notice that $a$ and $b$ terms correspond to $s$ - and $p$-wave contributions, respectively. Taking $m_{\tilde{f}} \sim m_{\mathrm{LSP}} \sim 100 \mathrm{GeV}$, the annihilation cross section given in eq. (11) is at most $3 \times 10^{-8} \mathrm{GeV}^{-2}$. Using this result in the inequality (10), we can see that the reheating temperature must be higher than about $100 \mathrm{MeV}$ even if $\left\langle v^{2}\right\rangle \sim 1$. If the average velocity is smaller than 1 , the constraint becomes more stringent, as we will see below.

\section{Thermalization of LSP}

In order to obtain the precise lowerbound on the reheating temperature $T_{R}$, we have to know $\left\langle v^{2}\right\rangle$, as well as the mass spectrum of the superparticles on which the annihilation cross section depends. First, let us discuss the averaged velocity of LSP, $\left\langle v^{2}\right\rangle$. Since LSP is mostly the bino, it loses its energy by scattering off the background fermions. In the model with the no-scale type boundary conditions, right-handed sleptons become the lightest among the sfermions, and hence LSP loses its energy mainly by scattering off the background electron (and $\mu$ and $\tau$, if the temperature is higher than their masses). If LSP is relativistic, the cross section for this process, $\sigma_{\text {scatt }}$, is estimated as

$$
\left\langle\sigma_{\text {scatt }} v_{\text {rel }}\right\rangle \simeq 128 \pi \alpha_{1}^{2} \frac{E_{\mathrm{LSP}}^{2} T_{R}^{2}}{m_{\tilde{e}_{R}}^{4} m_{\mathrm{LSP}}^{2}}
$$

where $E_{\mathrm{LSP}}$ is the energy of LSP, and $m_{\tilde{e}_{R}}$ the mass of the right-handed selectron.5 The energy loss rate for the relativistic LSP, $\Gamma_{\text {scatt }}^{\mathrm{R}}$, is given by

$$
\Gamma_{\mathrm{scatt}}^{\mathrm{R}} \simeq n_{e}\left\langle\sigma_{\mathrm{scatt}} v_{\mathrm{rel}}\right\rangle \frac{\Delta E_{\mathrm{LSP}}}{E_{\mathrm{LSP}}}
$$

where $n_{e}$ represents the number density of the background electron and $\Delta E_{\mathrm{LSP}}$ is the averaged energy loss of LSP in one scattering which is given by

$$
\Delta E_{\mathrm{LSP}} \simeq-12 E_{\mathrm{LSP}}\left(\frac{T_{R} E_{\mathrm{LSP}}}{m_{\mathrm{LSP}}^{2}}\right) .
$$

\footnotetext{
${ }^{3}$ This cross section is applied for $E_{\mathrm{LSP}} T_{R} \ll m_{\tilde{e}_{R}}^{2}$.
} 
Taking the ratio of the energy loss rate $\Gamma_{\text {scatt }}^{\mathrm{R}}$ to the expansion rate $H$ of the universe, we find

$$
\left.\frac{\Gamma_{\text {scatt }}^{\mathrm{R}}}{H}\right|_{E_{\mathrm{LSP}} \gg m_{\mathrm{LSP}}} \simeq 2 \times 10^{3}\left(\frac{E_{\mathrm{LSP}}}{10^{2} \mathrm{GeV}}\right)^{3}\left(\frac{T_{R}}{100 \mathrm{MeV}}\right)^{4}\left(\frac{100 \mathrm{GeV}}{m_{\tilde{e}_{R}}}\right)^{4}\left(\frac{100 \mathrm{GeV}}{m_{\mathrm{LSP}}}\right)^{4} .
$$

Thus, if $T_{R} \gtrsim$ a few $\times 10 \mathrm{MeV}$, the energetic LSP loses its energy through the scattering off thermal electrons efficiently for $m_{\tilde{e}_{R}} \sim m_{\mathrm{LSP}} \simeq O(100 / G E V)$, and becomes a nonrelativistic particle.

The non-relativistic LSP further loses its energy by scattering off background electrons. The averaged loss of the kinetic energy for the non-relativistic LSP in one scattering process, $\Delta \epsilon_{\mathrm{LSP}}$, is given by

$$
\Delta \epsilon_{\mathrm{LSP}} \simeq-\frac{20 \epsilon_{\mathrm{LSP}} T_{R}}{m_{\mathrm{LSP}}}\left(1-\frac{T_{R}}{\epsilon_{\mathrm{LSP}}}\right),
$$

where $\epsilon_{\mathrm{LSP}} \equiv E_{\mathrm{LSP}}-m_{\mathrm{LSP}}$ is the kinetic energy of LSP. As one can see in eq.(18), the LSP which has a kinetic energy larger than $\sim T_{R}$ tends to lose its energy through the scattering process, while LSP receives energy from the thermal bath if its energy is smaller than $\sim T_{R}$. Thus, if the scattering processes take place effectively, the averaged kinetic energy of LSP becomes $\sim T_{R}$, i.e. LSP goes into the kinetic equilibrium.

The energy loss rate $\Gamma_{\mathrm{scatt}}^{\mathrm{NR}}$ for the non-relativistic LSP is given by

$$
\Gamma_{\mathrm{scatt}}^{\mathrm{NR}} \simeq n_{e}\left\langle\sigma_{\mathrm{scatt}} v_{\mathrm{rel}}\right\rangle \times \frac{20 T_{R}}{m_{\mathrm{LSP}}} \simeq \frac{5760 \alpha_{1}^{2}}{\pi} \frac{T_{R}^{6}}{m_{\mathrm{LSP}} m_{\tilde{e}_{R}}^{4}} .
$$

The LSP goes into the kinetic equilibrium if the scattering rate $\Gamma_{\text {scatt }}^{\mathrm{NR}}$ is larger than the expansion rate of the universe. Taking $m_{\tilde{e}_{R}} \sim m_{\mathrm{LSP}}$, the ratio of $\Gamma_{\mathrm{scatt}}^{\mathrm{NR}}$ to the expansion rate of the universe, $H$, is given by

$$
\left.\frac{\Gamma_{\text {scatt }}^{\mathrm{NR}}}{H}\right|_{E_{\mathrm{LSP}} \sim m_{\mathrm{LSP}}} \simeq 4 \times 10^{3}\left(\frac{m_{\mathrm{LSP}}}{100 \mathrm{GeV}}\right)^{-5}\left(\frac{T_{R}}{100 \mathrm{MeV}}\right)^{4} .
$$

Thus, if the reheating temperature is higher than about $10 \mathrm{MeV}$, produced LSPs go into kinetic equilibrium as far as $m_{\mathrm{LSP}} \sim O(100) \mathrm{GeV}$. Furthermore, as we discussed in the previous section, the reheating temperature should be higher than at least $100 \mathrm{MeV}$ in

\footnotetext{
${ }^{4}$ Naively, it is expected that $\Delta \epsilon_{\mathrm{LSP}}$ is $\sim T_{R}$. However this order of the energy loss is cancelled out when the average is taken over angles of the incident particles and the actual energy loss is much smaller than the naive expectation.
} 
order to decrease the mass density of LSP sufficiently. Thus, we conclude that the produced LSPs go into kinetic equilibrium if we require that the mass density of the relic LSP should not overclose the universe. In this case, the averaged velocity is given by

$$
\left\langle v^{2}\right\rangle \simeq \frac{3 T_{R}}{m_{\mathrm{LSP}}}
$$

¿From this we easily see that the LSP abundance given in eq.(9) decreases as the reheating temperature gets higher. Thus, we obtain the lowerbound on the reheating temperature.

\section{Results}

Once we know the averaged velocity $\left\langle v^{2}\right\rangle$, we can calculate the annihilation cross section of LSP, and get the lowerbound on the reheating temperature after the decay of the Polonyi field. In this letter, we first solve RGEs based on the minimal SU(5) model with the no-scale boundary conditions, and determine the mass spectrum of the superparticles. We only investigate the parameter space which is not excluded by the experimental or theoretical constraints. The constraints which we use are as follows:

- Higgs bosons $H_{f}$ and $\bar{H}_{f}$ have correct vacuum expectation values.

- Perturbative picture is valid below the gravitational scale.

- LSP is neutral.

- Sfermions (especially, charged sleptons) have masses larger than the experimental lower limits [8].

- The branching ratio for $Z$-boson decaying into neutralinos is not too large [9].

Then, with the obtained mass spectrum of superparticles, we calculate the annihilation cross section and determine the lowerbound on the reheating temperature from the following equation;

$$
\left.\frac{H}{s\left\langle\sigma_{\mathrm{ann}} v_{\mathrm{rel}}\right\rangle}\right|_{T=T_{R}} \leq \frac{\rho_{c}}{s_{0}} \simeq 3.6 h^{2} \times 10^{-9} \mathrm{GeV} .
$$

In fig. 1 , we show the lowerbound on the reheating temperature in the $\tan \beta$ vs. $m_{\mathrm{LSP}}$ plane. In the figures, large or small $\tan \beta$ 's are not allowed since the Yukawa coupling

\footnotetext{
${ }^{5}$ By the numerical calculation we have checked, in fact, that the scattering rate given in eq. (19) is always larger than the expansion rate of the universe when the relic LSP does not overclose the universe. (See fig.2.)
} 
constant for the top quark or bottom quark blows up below the gravitational scale for such $\tan \beta$ 's. Furthermore, there also exists a lowerbound on the LSP mass. In the case where $\tan \beta \lesssim 20$, charged sfermions become lighter than the experimental limit if the LSP mass becomes lighter than $\sim 50 \mathrm{GeV}$. On the other hand, for the large $\tan \beta$ case, unless the bino mass is sufficiently large, the lightest charged slepton becomes LSP. (Remember that the dominant component of LSP is bino.) Thus, the lowerbound on $m_{\mathrm{LSP}}$ is obtained. As we can see, the reheating temperature should be larger than about $100 \mathrm{MeV}$, even for the case where $m_{\mathrm{LSP}} \sim 50 \mathrm{GeV}$. The constraint becomes more stringent as $m_{\mathrm{LSP}}$ increases, since the masses of the superparticles which mediate the annihilation of LSP becomes larger as the LSP mass increases.

If we translate the lowerbound on the reheating temperature into that of the Polonyi mass $m_{\phi}$, we obtain $m_{\phi} \gtrsim 100 \mathrm{TeV}$ (see eq.(2)). We can also see that the lowerbound is almost independent of $\tan \beta$. In fig. 2, We show the lowerbound on $T_{R}$ as a function of the LSP mass for $\tan \beta=10$, and $\mu_{H}>0$.

Here, we should comment on the accidental case where the annihilation process hits the Higgs pole in the $s$-channel. If the LSP mass is just half of the lightest Higgs boson mass, the LSP annihilation cross section is enhanced since LSP has small but nonvanishing fraction of higgsino component. If the parameters are well tuned, such a situation can be realized and the lowerbound of $T_{R}$ decreases to $O(10) \mathrm{MeV}$. However, we consider that such a scenario are very unnatural since a precise adjustment of the parameters is required in order to hit the Higgs pole.9

\section{Conclusions}

In this letter, we have obtained the lowerbound on the reheating temperature due to the decay of the Polonyi field in a framework of the no-scale type supergravity model. As a result, we have seen that the Polonyi mass should be larger than about $100 \mathrm{TeV}$ which may raise a new fine-tuning problem [10].

We have assumed the minimal SUSY GUT model in the present analysis. However, the main conclusion is not changed as far as LSP is mostly the bino, because the minimum value of the lowerbound $\left(T_{R} \simeq 100 \mathrm{MeV}\right)$ is obtained when the mass of the selectron takes the experimentally allowed lower limit.

\footnotetext{
${ }^{6}$ In the case where the annihilation process hits the pole of heavier Higgs bosons, the cross section is not enhanced so much, since the widths of the heavier Higgs bosons are quite large.
} 
We have assumed that LSP is stable so far. However, if we introduce $R$-parity violation, LSP becomes unstable and the allowed $T_{R}$ is as low as a few MeV. It is also the case if we assume a very light LSP such as a neutral higgsino [1] or an axino [1] whose masses are less than about $100 \mathrm{MeV}$.

\section{Acknowledgement}

Two of the authors (T.M. and T.Y.) would like to thank M. Yamaguchi for useful discussions in the early stage of this work. 


\section{References}

[1] G.D. Coughlan, W. Fischler, E.W. Kolb, S. Raby and G.G. Ross, Phys. Lett. B131 (1983) 59.

[2] T. Banks, D.B. Kaplan and A.E. Nelson, Phys. Rev. D49 (1994) 779;

B. de Carlos, J.A. Carlos, F. Quevedo and E. Roulet, Phys. Lett. B318 (1993) 447.

[3] E. Cremmer, S. Ferrara, L. Grardello and A. van Proeyen, Nucl. Phys. B212 (1983) 413.

[4] J. Ellis, D.V. Nanopoulos and M. Quiros, Phys. Lett. B174 (1986) 176.

[5] T. Moroi, M. Yamaguchi and T. Yanagida, Phys. Lett. B342 (1995) 105.

[6] J. Eliis, C. Kounnas and D.V. Nanopoulos, Nucl. Phys. B241 (1984) 406;

H. Murayama, H. Suzuki, T. Yanagida and J. Yokoyama, Phys. Rev. D50 (1994) 2356.

[7] K.A. Olive and M. Srednicki, Phys. Lett. B230 (1989) 78.

[8] Particle Data Group, Phys. Rev. D50 (1994) 1173.

[9] L3 Collaboration, Phys. Lett. B350 (1995) 109.

[10] G.A. Diamandis, J. Ellis, A.B. Lahanas and D.V. Nanopoulos, Phys. Lett. B173 (1986) 303;

P. Binetruy and M.K. Gaillard, Phys. Lett. B168 (1986) 347.

[11] K. Rajagopal, M.S. Turner and F. Wilczek, Nucl. Phys. B358 (1991) 447;

T. Goto and M. Yamaguchi, Phys. Lett. B276 (1992) 103;

E.J. Chun, J.E. Kim and H.P. Nilles, Phys. Lett. B287 (1992) 123. 


\section{Figure Caption}

fig. 1

Lowerbound on $T_{R}$ is shown in $\tan \beta$ vs. $m_{\mathrm{LSP}}$ plane. The meaning of each marks is as follows; $\circ: 100 \mathrm{MeV} \leq T_{R} \leq 500 \mathrm{MeV}, \times: 500 \mathrm{MeV} \leq T_{R} \leq 1 \mathrm{GeV}, \square: 1 \mathrm{GeV} \leq$ $T_{R} \leq 5 \mathrm{GeV},+: 5 \mathrm{GeV} \leq T_{R} \leq 10 \mathrm{GeV}, \diamond: 10 \mathrm{GeV} \leq T_{R} \leq 50 \mathrm{GeV}$. The sign of the SUSY-invariant Higgs mass $\mu_{H}$ is taken to be (a) $\mu_{H}>0$, and (b) $\mu_{H}<0$.

fig. 2

Lowerbound on $T_{R}$ is shown as a function of $m_{\mathrm{LSP}}$. Here, we take $\tan \beta=10$ and $\mu_{H}>0$ in the solid line. Furthermore, the temperature at which the relation $\Gamma_{\text {scatt }}=H$ realizes is also shown in dashed line. 


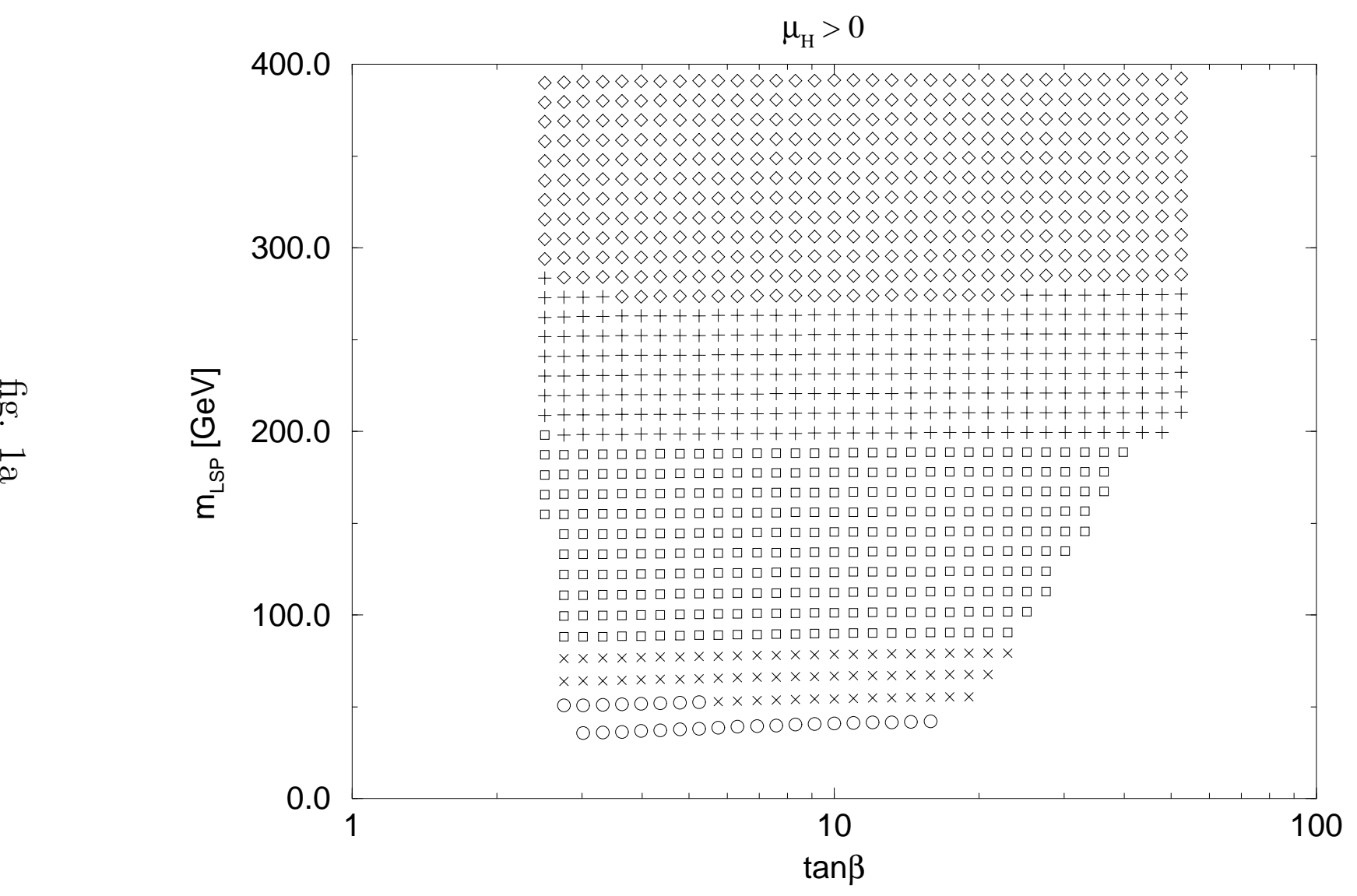




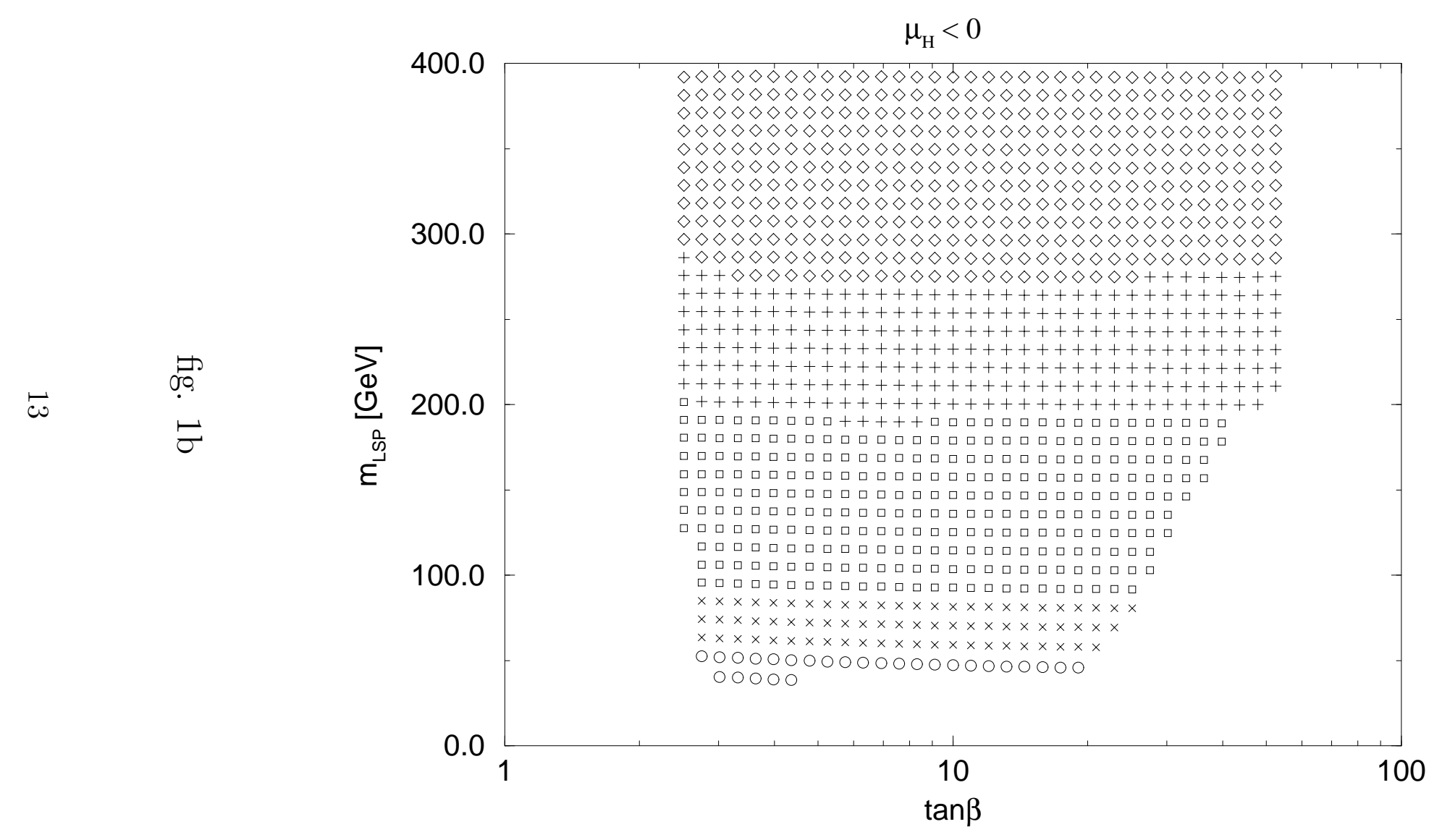




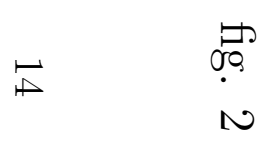

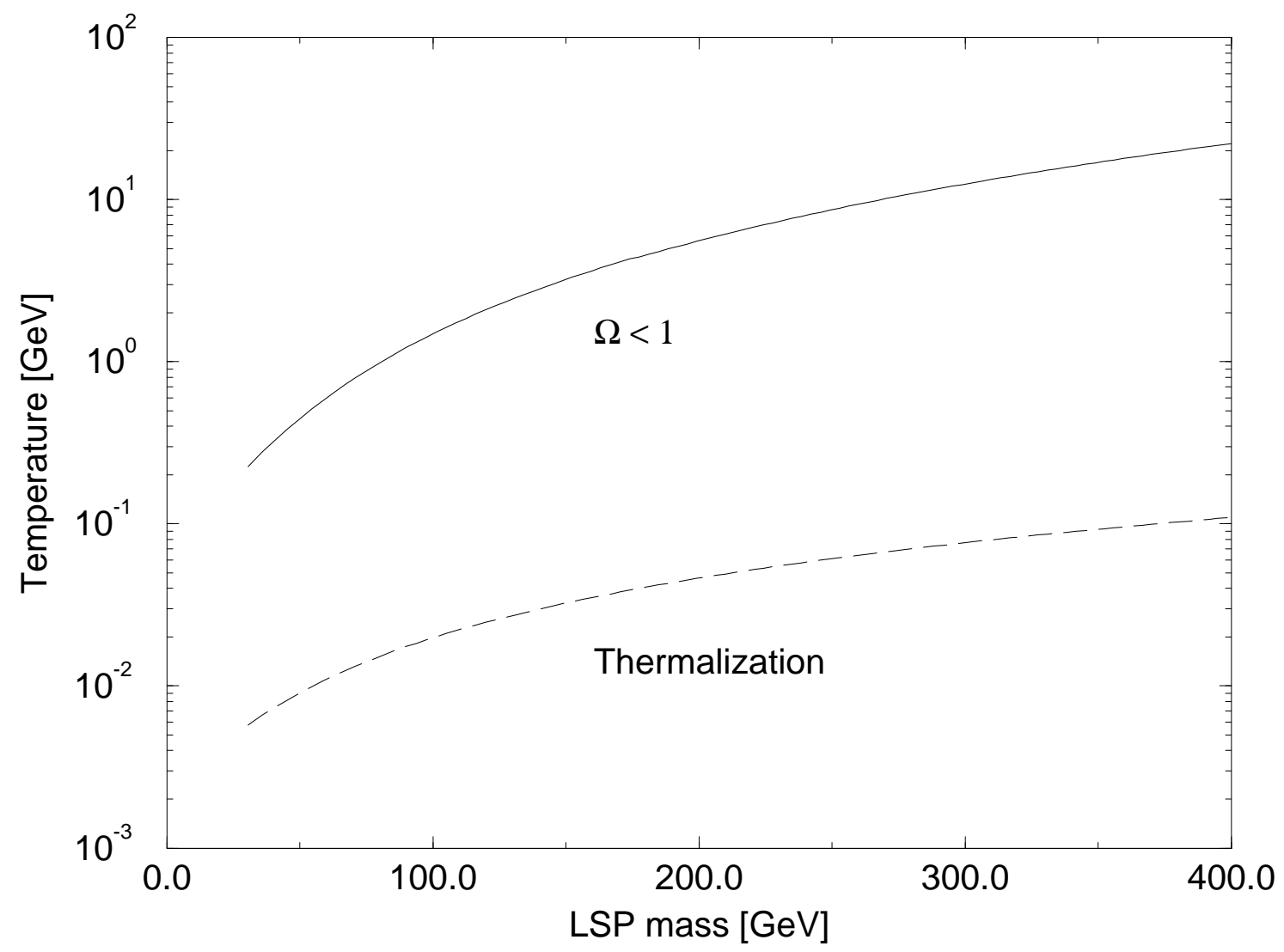

